\title{
Evaluation of the serum ceruloplasmin level before and after non-surgical periodontal therapy in patients with chronic periodontitis
}

\author{
Abdulkarem A. Mohammed ${ }^{1}$, Jilan M. Youssef ${ }^{2}$, Shereen S. Metwally ${ }^{3}$, Mohamed M. Anees ${ }^{2}$ \\ 1Department of Periodontology, Faculty of Dentistry, Thamar University, Dhamar 009676, Yemen. \\ 2Departemnt of Oral Medicine and Periodontology, Faculty of Dentistry, Mansoura University, Mansoura 35516, Egypt.
}

${ }^{3}$ Department of Clinical Pathology, Faculty of Medicine, Mansoura University, Mansoura 35516, Egypt.

Correspondence to: Dr. Abdulkarem A. Mohammed, Department of Periodontology, Faculty of Dentistry, Thamar University, Dhamar 009676, Yemen. E-mail: alshari19@yahoo.com

How to cite this article: Mohammed AA, Youssef JM, Metwally SS, Anees MM. Evaluation of the serum ceruloplasmin level before and after non-surgical periodontal therapy in patients with chronic periodontitis. Stomatological Dis Sci2018;2:3. http://dx.doi.org/10.20517/2573-0002.2017.15

Received: 20 Sep 2017 First Decision: 8 Feb 2018 Revised: 8 Mar 2018 Accepted: 8 Mar 2018 Published: 14 Mar 2018

Science Editors: Nikolaos G. Nikitakis, Dimitrios Andreadis Copy Editor: Jun-Yao Li Production Editor: Cai-Hong Wang

\begin{abstract}
Aim: This study aimed to evaluate the serum ceruloplasmin (CP) level after non-surgical periodontal therapy in chronic periodontitis patients.
\end{abstract}

Methods: A prospective controlled study was conducted on 80 subjects. The study populations were divided into 2 groups: group 1 included chronic periodontitis patients (study group, $n=40$ ), and group 2 included periodontally healthy subjects (control group, $n=40$ ). Blood sample and periodontal clinical parameters, including periodontal pocket depth, clinical attachment level and bleeding on probing, were performed at baseline for both groups. All of the patients with chronic periodontitis (study group) received meticulous scaling and root planing twice weekly for 2 weeks. Four weeks after treatment, the second blood sample and reevaluation of clinical periodontal parameters were done.

Results: Baseline serum CP level was significantly higher in chronic periodontitis patients (study group) compared to healthy subjects (control group) $(P<0.001)$. Concerning the chronic periodontitis group, four weeks after non-surgical periodontal therapy, the mean value of serum CP concentration was significantly decreased $(P<0.001)$.

Conclusion: Non-surgical periodontal therapy has a reducing effect on the serum CP level in chronic periodontitis patients. Serum CP level represents a potential biomarker indicator of the chronic periodontitis disease.

Keywords: Biomarkers, ceruloplasmin, chronic periodontitis, non-surgical periodontal therapy

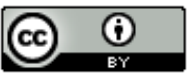

(C) The Author(s) 2018. Open Access This article is licensed under a Creative Commons Attribution 4.0 International License (https://creativecommons.org/licenses/by/4.0/), which permits unrestricted use, sharing, adaptation, distribution and reproduction in any medium or format, for any purpose, even commercially, as long as you give appropriate credit to the original author(s) and the source, provide a link to the Creative Commons license, and indicate if changes were made. 


\section{INTRODUCTION}

Periodontitis is a multifactorial inflammatory disease caused by interactions between periodontal microflora and host response ${ }^{[1]}$. Progressive periodontal destruction is an important sequela of periodontitis that can lead to the loosening and subsequent loss of teeth ${ }^{[2]}$.

The most common clinical features of chronic periodontitis are the loss of connective tissue fibers, alveolar bone destruction, the formation of periodontal pockets, and gingival inflammation. Additional signs and symptoms of a chronic periodontal disease are the accumulation of supra- and sub-gingival calculus, gingival enlargement or recession, bleeding, furcation involvement, the mobility of the teeth, and bad odors ${ }^{[3]}$.

The aim of non-surgical periodontal treatment (NPT) is the elimination of bacterial deposits which adhere to tooth surfaces, primarily by means of dental plaque control performed by the patient, in addition to dental scaling and root surface debridement performed by the dentist ${ }^{[4]}$. Systematic reviews and meta-analysis support the effect of NPT for controlling periodontal disease by reducing inflammation ${ }^{[5]}$.

Clinical and radiographic assessment is used as traditional measures for diagnosis of periodontal diseases. These traditional measures give only information about the existing disease but limited to predict in early diagnosis of disease ${ }^{[6]}$. Therefore, advances in oral and periodontal disease diagnostic research are moving toward methods, whereby, periodontal risk can be identified and quantified by objective measures like biomarkers ${ }^{[7]}$. One of these biomarkers that can be detected in several inflammatory conditions is ceruloplasmin $(\mathrm{CP})^{[8]}$.

$\mathrm{CP}$ can be defined as a copper-containing glycoprotein with a molecular weight of $132 \mathrm{kDa}$. It is synthesized mainly in the liver and has many functions; such as an antioxidant, a ferroxidase, for copper transport, antiinflammation, and proinflammatory activities ${ }^{[9,10]}$. Ferroxidase activity is one of the important actions of CP that is involved in oxidation of iron from the $\mathrm{Fe}^{2+}$ ion state to the $\mathrm{Fe}^{3+}$ ion state, which represents a crucial step for adequate trans-cellular ionic transport ${ }^{[11]}$.

In addition, $\mathrm{CP}$ is an acute phase reactant, and its serum concentration is increased during inflammation, infection, and trauma largely as a result of increased gene transcription in hepatocytes mediated by the inflammatory cytokines ${ }^{[12]}$. In addition, CP plays an important role in reducing oxidative stress by its ability to scavenge superoxide anion radicals. Furthermore, it has been proved that $\mathrm{CP}$ causes priming of the neutrophils in localized aggressive periodontitis (LAgP). Serum CP levels were increased among subjects with LAgP compared to serum samples from matched healthy donors ${ }^{[13]}$.

This study hypothesized that serum CP levels would be increased in patients with chronic periodontitis. Many previous studies reported that $\mathrm{CP}$ was a useful biomarker for many inflammatory diseases as well as in aggressive and chronic periodontitis ${ }^{[8,11]}$. However, there is a lack of reports about the relationship between $\mathrm{CP}$ and chronic periodontitis. Therefore, this study aimed to evaluate the serum CP level after non-surgical periodontal therapy in the chronic periodontitis patients.

\section{METHODS}

This study was conducted on 80 non-smokers systemically healthy subjects. All subjects were selected from the Oral Medicine and Periodontology Department, Faculty of Dentistry, Mansoura University, Egypt. The participants were categorized into two groups based on periodontal health. Group 1: chronic periodontitis patients as a study group comprised of 40 subjects ( 25 males and 15 females), with a mean age of 41.28 years (range 30 to 50 years); they were diagnosed with chronic periodontitis. Group 2: control group with healthy periodontium comprised of 40 subjects ( 26 males and 14 females), with a mean age of 39.67 years (range 34 to 48 years). 
Patients included in our study (study group) were classified as having moderate (20 patients) or severe (20 patients) chronic periodontitis. Patients were classified according to the criteria mentioned by American Academy of Periodontology workshop in 1999 in which moderate and severe chronic periodontitis case have clinical attachment loss of 3-4 $\mathrm{mm}$, and more than $5 \mathrm{~mm}$, respectively ${ }^{[14]}$.

Written informed consent was obtained from all subjects at the baseline. Patients were excluded from the study if they had any systemic diseases that could influence the outcome of therapy, patients receivd surgical periodontal treatment in the last six months, acute inflammatory conditions that might affect serum $\mathrm{CP}$ level, pregnancy, smokers, and immunosuppressive chemotherapy.

A careful clinical examination, patient motivation about the benefit of the treatment and plaque control, detailed instruction about the therapeutic program, a documented case history, a panoramic radiograph, and the first blood sample were performed for all study populations at the baseline.

Following this, the chronic periodontitis patients received full-mouth scaling and root planing under local anesthesia. Scaling was done using piezoelectric ultrasonic scalers, and root planing was performed using specific Gracey curettes. No antibiotics were prescribed following this treatment. Oral hygiene instruction was provided. Scaling and root planing therapy was repeated twice weekly for two weeks. After four weeks of treatment, the second serum samples were taken.

The following parameters: bleeding on probing index (BOP), probing pocket depth (PPD) and clinical attachment level (CAL) were measured at the beginning of the study in both groups and four weeks after treatment in the study group. These clinical parameters were recorded using a Williams' periodontal probe (Hu-Friedy, Chicago, IL, USA).

Blood samples were withdrawn from the antecubital vein under aseptic condition. The blood samples were then sent to the Immunology Department of Clinical Pathology, Faculty of Medicine, Mansuora University. The samples were centrifuged at $2000 \mathrm{rpm}$ for $10 \mathrm{~min}$. Serum samples were collected and stored in Eppendorf tube at $-20{ }^{\circ} \mathrm{C}$ until the time of assay.

Serum CP level was analyzed by using Human CP ELISA Kit (Assaypro LLC, USA) which is designed for detection of human $\mathrm{CP}$ in plasma and serum samples.

\section{Statistical analysis}

The data was analyzed using the IBM SPSS software package version 20.0. (Armonk, NY: IBM Corp). Qualitative data were described using number and percent. The Kolmogorov-Smirnov test was used to verify the normality of the distribution. Quantitative data were described using range (minimum and maximum), mean, standard deviation, and median. The significance of the results was judged at the $5 \%$ level.

\section{RESULTS}

\section{Demographic data and clinical parameters assessment}

The age of the subjects in the control group ranged from 34.0 to 48.0 years with mean and standard deviation (SD) of $39.67 \pm 3.28$ years. Twenty-six (65.0\%) were male and 14 (35.0\%) were female.

However, the age of the patients in the study group ranged from 30 to 50 years with mean and SD of $41.28 \pm$ 6.13 years. Twenty-five (62.5\%) were male and $15(37.5 \%)$ were female [Table 1]. Demographic data of all study populations are illustrated in Table 1. There was no significant difference between the two groups regarding age and gender. 
Table 1. Comparison between the two studied groups according to demographic data

\begin{tabular}{lccc}
\hline & Study group $(\boldsymbol{n}=\mathbf{4 0})$ & Control group $(\boldsymbol{n}=\mathbf{4 0})$ & $\boldsymbol{P}$ \\
\hline Gender, $n(\%)$ & $25(62.5)$ & $26(65.0)$ & 0.816 \\
Male & $15(37.5)$ & $14(35.0)$ & \\
Female & & & \\
Age (years) & $30.0-50.0$ & $34.0-48.0$ & \\
Range & $41.28 \pm 6.13$ & $39.67 \pm 3.28$ & \\
Mean 4 SD & &
\end{tabular}

Table 2. Comparison between the two studied groups according to different parameters

\begin{tabular}{llccl}
\hline & & Study group $(\boldsymbol{n}=\mathbf{4 0})$ & Control group $(\boldsymbol{n}=\mathbf{4 0})$ & \multicolumn{1}{c}{$\boldsymbol{P}$} \\
\hline Ceruloplasmin level & Before & $42.62 \pm 12.57$ & $21.59 \pm 3.95$ & $<0.001^{*}$ \\
& After & $26.40 \pm 7.48$ & $21.59 \pm 3.95$ & $0.001^{*}$ \\
PPD & Before & $4.62 \pm 0.97$ & $0.98 \pm 0.60$ & $<0.001^{*}$ \\
& After & $3.71 \pm 0.69$ & $0.98 \pm 0.60$ & $<0.001^{*}$ \\
CAL & Before & $5.47 \pm 1.21$ & $0.0 \pm 0.0$ & $<0.001^{*}$ \\
& After & $4.53 \pm 1.04$ & $0.0 \pm 0.0$ & $<0.001^{*}$ \\
BOP & Before & $4.61 \pm 0.38$ & $0.09 \pm 0.12$ & $<0.001^{*}$ \\
& After & $0.32 \pm 0.33$ & $0.09 \pm 0.12$ & $<0.001^{*}$
\end{tabular}

*Statistically significant at $P \leq 0.05$. PPD: probing pocket depth; CAL: clinical attachment level; BOP: bleeding on probing index

Regarding clinical parameters (PPD, CAL, and BOP), the values of the mean and SD were $4.62 \pm 0.97,5.47$ \pm 1.21 and $4.61 \pm 0.38$ respectively. There was a significant difference between the chronic periodontitis group and the healthy control group at the baseline and after non-surgical periodontal therapy $(P<0.001)$. Furthermore, the mean and SD of the clinical parameters (PPD, CAL, and BOP) in the chronic periodontitis group after treatment were $3.71 \pm 0.69,4.53 \pm 1.04$ and $0.32 \pm 0.33$ respectively. These values showed a significant reduction after the treatment compared to its values at the baseline [Table 2].

\section{Serum CP level analysis}

The mean values of serum CP level in the study group and the control group at the baseline were $42.62 \pm 12.57$ and $21.59 \pm 3.95$ respectively. These results showed that there was a significant difference between the two groups $(P<0.001)$.

Regarding chronic periodontitis patients (study group), the mean value of serum CP level after treatment was significantly reduced compared to its value at the baseline [Table 3].

\section{DISCUSSION}

This study conducted on 80 individuals, divided into 2 groups. Forty patients suffering from chronic periodontitis served as a study group while the control group was represented by 40 periodontally healthy subjects.

Patients suffering from any acute systemic inflammatory conditions were excluded because the level of CP may be affected as $\mathrm{CP}$ is an acute phase reactant protein, where its serum concentrations rise significantly during acute inflammation ${ }^{[15]}$.

Additionally, we excluded those who were suffering from systemic diseases, such as, diabetes because of the clear effect of diabetes on the periodontal condition and therapy ${ }^{[16]}$. CP also has indirect effect on the $\beta$ cells producing insulin in the pancreas by iron accumulation in the cells ${ }^{[17]}$. Patients suffering from cardiovascular diseases, neurodegenerative disorder, chronic infections (e.g., tuberculosis and degenerative diseases), cancers, and hepatic diseases were excluded from this study because these conditions interfere with initiation, progression, and treatment outcomes of periodontal diseases and affect the CP concentration ${ }^{[18-20]}$. 
Table 3. Correlation between ceruloplasmin and periodontal (PPD, CAL and BOP) in study group

\begin{tabular}{lccc}
\hline Periodontal parameters & & \multicolumn{2}{c}{ Ceruloplasmin in study group } \\
\cline { 2 - 4 } & & Before & After \\
\hline PPD & $r$ & $0.363^{*}$ & 0.134 \\
& $P$ & $0.021^{\star}$ & 0.410 \\
CAL & $r$ & $0.393^{\star}$ & 0.117 \\
& $P$ & $0.012^{\star}$ & 0.471 \\
BOP & $r$ & 0.005 & 0.310 \\
& $P$ & 0.974 & 0.052 \\
\hline
\end{tabular}

*Statistically significant at $P \leq 0.05$. PPD: probing pocket depth; CAL: clinical attachment level; BOP: bleeding on probing index

The clinical periodontal parameters (PPD, CAL, BOP) were recorded at the baseline for all of the tested populations (both study and control groups), and after treatment regarding chronic periodontitis patients. Serum samples were used to investigate the level of CP because it is mainly synthesized by the liver and then secreted into the blood. CP is also the major copper-carrying protein in the blood ${ }^{[21]}$.

As would be expected, there was a significant elevation in PPD, CAL and BOP values of chronic periodontitis patients as compared to healthy subjects at the baseline.

Regarding the chronic periodontitis patients, the mean values of periodontal indices (PPD, CAL, BOP) after scaling and root planing (SRP) were found to be significantly decreased versus their level at baseline. These results are in agreement with Chan and Lai ${ }^{[22]}$ and De Micheli et al. ${ }^{[23]}$ who found significant reduction in probing pocket depth and bleeding on probing following non-surgical therapy. Also, Darby et al. ${ }^{[24]}$ showed that SRP resulted in improvement in the clinical attachment loss measurements mean values. However, this significant improvement in the measured parameters still displayed significant difference compared to healthy group.

Regarding the chronic periodontitis patients, the improvement in clinical parameters after SRP may be due to resolution of the inflammatory response and the cessation of periodontal disease destruction. These outcomes result in a relative gain of clinical attachment and a reduction of the probing $\operatorname{depth}^{[25,26]}$.

At the baseline, the serum CP level of the study group was statistically significantly higher than that of control subjects. These results disagreed with that of Harshavardhana et al ${ }^{[8]}$ as they found non-significant changes in levels of serum $\mathrm{CP}$ in the chronic periodontitis patients in comparison to the healthy subjects. They attributed this result to the small sample size studied. Varghese et al. ${ }^{[27]}$ found that the CP levels in GCF and the tissues in aggressive periodontitis patients were significantly higher when compared to the healthy subjects.

The higher level of the serum CP regarding chronic periodontitis patients may be due to the role of CP as a proinflammatory mediator, acute phase protein and an anti-inflammatory function which is carried by an antioxidant, bactericidal, and ferroxidase activities ${ }^{[28]}$. Additionally, this anti-inflammatory function may be due to the ability of $\mathrm{CP}$ to limit the oxidative stress by scavenging superoxide anion radicals $\left(\mathrm{O}_{2}\right)$ which can be generated by polymorphonuclear cells (PMNs) during inflammation that may lead to tissue damage ${ }^{[29]}$. Moreover, the higher level of CP during inflammation could be attributed to its role as a protease inhibitor $^{[25]}$.

Iwata et al.$^{[13]}$ demonstrated that $\mathrm{CP}$ expression and activity were significantly higher in PMNs and sera from subjects diagnosed with LAgP compared to healthy subjects. They reported that $\mathrm{CP}$ causes priming of the PMNs in LAgP; thus, PMNS can produce a faster and greater response to secondary challenges and present a "primed" phenotype ${ }^{[30]}$. 
Concerning the present study, it was found that SRP significantly reduced the level of serum CP in chronic periodontitis when compared to its value at the baseline. This reduction in the CP level at the end of the study can be explained by the effect of SRP on removal of local factors; thus, it can decrease the inflammatory response and consequently decrease the mediators that stimulate acute phase protein production ${ }^{[23]}$. We were not able to compare the results of the present study about the effect of SRP on serum level of CP in the chronic periodontitis patients with other studies because we did not find any previous studies evaluating the effect of SRP on the CP level.

Even with the limitations of this study, we can conclude that non-surgical periodontal therapy has a reducing effect on the serum CP level in chronic periodontitis patients. In addition, serum CP levels represent a potential biomarker of the chronic periodontitis disease.

\section{DECLARATIONS}

\section{Acknowledgments}

The authors would like to acknowledge the efforts of Dr. Jon Wanger in refining the final manuscript.

\section{Authors' contributions}

Manuscript preparation, practical part and initial writing: Mohammed AA

Supervising in clinical and manuscript review: Youssef JM, Anees MM

Supervising of Immunological and Laboratory work: Metwally SS

\section{Data source and availability}

All data are stored in the liberary of Faculty of Dentistry, Mansoura University.

\section{Financial support and sponsorship}

None.

\section{Conflicts of interest}

There are no conflicts of interest.

\section{Patient consent}

Written informed consent was obtained from all subjects at the baseline.

\section{Ethics approval}

The procedures followed were in accordance with the rules of the Ethics Committee of the Faculty of Dentistry, Mansoura University and Medical Ethics in dealing with children and parents. All steps of infection control and the sterilization protocol were followed.

\section{Copyright}

(c) The Author(s) 2018.

\section{REFERENCES}

1. de Queiroz AC, Taba M Jr, O’Connell PA, da Nóbrega PB, Costa PP, Kawata VK, Trevisan GL, Novaes AB Jr, de Souza SL, Palioto DB, Grisi MF. Inflammation markers in healthy and periodontitis patients: a preliminary data screening. Braz Dent J 2008;19:3-8.

2. Tonetti MS, Van Dyke TE; Working group 1 of the joint EFP/AAP workshop. Periodontitis and atherosclerotic cardiovascular disease: consensus report of the Joint EFP/AAP Workshop on Periodontitis and Systemic Diseases. J Clin Periodontol 2013;40:S24-9.

3. Dentino A, Lee S, Mailhot J, Hefti AF. Principles of periodontology. Periodontol 2000 2013;61:16-53.

4. Claffey N, Polyzois I, Ziaka P. An overview of nonsurgical and surgical therapy. Periodontol 2000 2004;36:35-44.

5. Van der Weijden GA, Timmerman MF. A systematic review on the clinical efficacy of subgingival debridement in the treatment of chronic 
periodontitis. J Clin Periodontol 2002;29 Suppl 3:55-71; discussion 90-1.

6. Gamonal J, Bascones A, Acevedo A, Blanco E, Silva A. Apoptosis in chronic adult periodontitis analyzed by in situ DNA breaks, electron microscopy, and immunohistochemistry. J Periodontol 2001;72:517-25.

7. Taba M, Kinney J, Kim AS, Giannobile WV. Diagnostic biomarkers for oral and periodontal diseases. Dent Clin North Am 2005;49:551-71

8. Harshavardhana B, Rath SK, Mukherjee M. Evaluation of serum ceruloplasmin in aggressive and chronic periodontitis patients. $J$ Indian Soc Periodontol 2013;17:333-7.

9. Healy J, Tipton K. Ceruloplasmin and what it might do. J Neural Transm (Vienna) 2007;114:777-81.

10. Shukla N, Maher J, Masters J, Angelini GD, Jeremy JY. Does oxidative stress change ceruloplasmin from a protective to a vasculopathic factor? Atherosclerosis 2006;187:238-50.

11. Inoue K, Sakano N, Ogino K, Sato Y, Wang DH, Kubo M, Takahashi H, Kanbara S, Miyatake N. Relationship between ceruloplasmin and oxidative biomarkers including ferritin among healthy Japanese. J Clin Biochem Nutr 2013;52:160-6.

12. Gitlin JD. Transcriptional regulation of ceruloplasmin gene expression during inflammation. J Biol Chem 1988;263:6281-7.

13. Iwata T, Kantarci A, Yagi M, Jackson T, Hasturk H, Kurihara H, Van Dyke TE. Ceruloplasmin induces polymorphonuclear leukocyte priming in localized aggressive periodontitis. J Periodontol 2009;80:1300-6.

14. Armitage GC. Development of a classification system for periodontal diseases and conditions. Ann Periodontol 1999;4:1-6.

15. Schaefer M, Gitlin JD. Genetic disorders of membrane transport. IV. Wilson's disease and Menkes disease. Am J Physiol 1999;276:G311-4.

16. Bullon P, Newman HN, Battino M. Obesity, diabetes mellitus, atherosclerosis and chronic periodontitis: a shared pathology via oxidative stress and mitochondrial dysfunction? Periodontol 2000 2014;64:139-53.

17. Gonzalez-Cuyar LF, Perry G, Miyajima H, Atwood CS, Riveros-Angel M, Lyons PF, Siedlak SL, Smith MA, Castellani RJ. Redox active iron accumulation in aceruloplasminemia. Neuropathology 2008;28:466-71.

18. Iacopino AM, Cutler CW. Pathophysiological relationships between periodontitis and systemic disease: recent concepts involving serum lipids. J Periodontol 2000;71:1375-84.

19. Gitlin JD. Transcriptional regulation of ceruloplasmin gene expression during inflammation. J Biol Chem 1988;263:6281-7.

20. Nobili V, Siotto M, Bedogni G, Ravà L, Pietrobattista A, Panera N, Alisi A, Squitti R. Levels of serum ceruloplasmin associate with pediatric nonalcoholic fatty liver disease. J Pediatr Gastroenterol Nutr 2013;56:370-5.

21. Gaware V, Kotade K, Dhamak K, Somawanshi S. Ceruloplasmin its role and significance: a review. Int J Biomed Res 2010;1:153-62.

22. Chan Y, Lai CH. Bactericidal effects of different laser wavelengths on periodontopathic germs in photodynamic therapy. Lasers Med Sci 2003;18:51-5.

23. De Micheli G, de Andrade AK, Alves VT, Seto M, Pannuti CM, Cai S. Efficacy of high intensity diode laser as an adjunct to non-surgical periodontal treatment: a randomized controlled trial. Lasers Med Sci 2011;26:43-8.

24. Darby IB, Hodge PJ, Riggio MP, Kinane DF. Clinical and microbiological effect of scaling and root planing in smoker and non-smoker chronic and aggressive periodontitis patients. J Clin Periodontol 2005;32:200-6.

25. Carvalho LH, D’Avila GB, Leao A, Haffajee AD, Socransky SS, Feres M. Scaling and root planing, systemic metronidazole and professional plaque removal in the treatment of chronic periodontitis in a Brazilian population. I. clinical results. J Clin Periodontol 2004;31:1070-6.

26. Tüter G, Kurtiş B, Serdar M, Aykan T, Okyay K, Yücel A, Toyman U, Pinar S, Cemri M, Cengel A, Walker SG, Golub LM. Effects of scaling and root planing and sub-antimicrobial dose doxycycline on oral and systemic biomarkers of disease in patients with both chronic periodontitis and coronary artery disease. J Clin Periodontol 2007;34:673-81.

27. Varghese JM, Bhat V, Bhat GS, Rao N. Evaluation of glutathione-s-transferase and ceruloplasmin levels in gingival crevicular fluid and gingival tissue as diagnostic markers for chronic periodontitis. Adv Biosci Biotechnol 2012;3:437-41.

28. Kotrikadze N, Zibzibadze M, Ramishvili L, Chigogidze T, Managadze L. Protective function of ceruloplasmin and its action on erythrocytes in men with prostate tumors. Eur Med Health Pharm J 2011;2:180-8.

29. Dutra F, Ciriolo MR, Calabrese L, Bechara EJ. Aminoacetone induces oxidative modification to human plasma ceruloplasmin. Chem Res Toxicol 2005; 18:755-60.

30. Kmiec Z. Cooperation of Liver Cells in Health and Disease. Springer Science \& Business Media; 2001. p. 86-92. 